\title{
COUPLED NEURONAL OSCILLATORS IN THE SNAIL LYMNAEA STAGNALIS: ENDOGENOUS CELLULAR PROPERTIES AND NETWORK INTERACTIONS
}

\author{
By M. EGELHAAF" $\uparrow$ AND P. R. BENJAMIN \\ School of Biological Sciences, University of Sussex, Falmer, Brighton \\ BNI 9QG, U.K. and Lehrstuhl für Biokybernetik der Universität, Auf \\ der Morgenstelle 28, D-7400 Tübingen, F.R.G.
}

(Received 24 March 1982-Accepted 2 September 1982)

\begin{abstract}
S U M MAR Y
The role of endogenous cellular properties and network interactions due to electrotonic coupling were investigated in two bilateral populations of 2-7 peripheral neurones ('Peripheral Bursters') in the snail Lymnaea stagnalis.

1. These cells are endogenously capable of bursting. Their burst frequency does not depend on the level of steady membrane polarization. Short hyperpolarizing current pulses injected during the bursting cycle induce phase advance and no phase delay in subsequent cycles, the phase advance being a function of the phase of stimulus application. Phase response and inter-burst interval curves have been constructed for short hyperpolarizing current pulses. Their shape depends on the intensity and sign of tonically injected current. This property of Peripheral Bursters is one reason for the independence of period duration from membrane polarization.

2. Coordination of burst activity of Peripheral Bursters has been studied as a function of coupling strength: whereas homolateral cells are relatively strongly electrotonically coupled and always burst synchronously, thus forming a single oscillatory unit, heterolateral cells are only weakly electrotonically coupled. Accordingly, they show more variable, though coordinated, patterns of activity. Most commonly, periods with 1: 1 entrainment alternate with periods with 1:2 entrainment. The duration and frequency of occurrence of either mode of entrainment are highly variable in different preparations.
\end{abstract}

\section{INTRODUCTION}

Pattern generation and the coordination of different patterns of activity within nervous networks have been studied intensively during the last decades. To understand how neuronal patterns are generated, it is necessary to assess both the role of endogenous properties of single neurones and the role of network interactions (Selverston, 1980). This however, has proved to be difficult in many systems which are most interesting from a behavioural point of view because of their complexity. Therefore,

† Present address: M.P.I. für Biologische Kybernetik, Spemannstr. 38, D-7400 Tübingen, Federal Republic of Germany.

Key words: Neuronal oscillators, electrotonic coupling, burst coordination. 
investigators interested in the mechanisms underlying neuronal pattern generatic have been concentrating on relatively simple 'model systems', such as the stomatogastric nervous system (Selverston, Russel, Miller \& King, 1976) and the cardiac ganglion (Tazaki \& Cooke, 1979) of crustaceans, leech swimming (Stent et al. 1978 ) or snail feeding (Benjamin \& Rose, 1980).

In this study a small network of electrotonically coupled peripheral bursting neurones ('Peripheral Bursters'), forming two bilateral clusters of cells on the buccal mass of the pond snail, Lymnaea stagnalis, is investigated. With only 2-7 cells, the system of Peripheral Bursters on each side of the buccal mass is probably one of the smallest neuronal networks that can be isolated from any other nervous influence and still maintains its rhythmic burst activity. Under certain conditions single Peripheral Bursters can be isolated and their endogenous bursting capability established. Thus, the requirement to characterize the intrinsic properties of single cells separately from network interactions can be met. This neuronal network, therefore, provides a good model system for studying the contribution to pattern generation of both endogenous cellular properties and network interactions due to electrotonic coupling.

The intrinsic properties of Peripheral Bursters and, in particular, factors determining their burst frequency have been analysed mainly with techniques derived from oscillator theory (Pavlidis, 1973). Emphasis is laid on those properties which differ in some respect from those of other neuronal oscillators. Network interactions among Peripheral Bursters have been studied under two different coupling conditions. This has been possible because homo- and heterolateral cells are electrotonically coupled with different coupling strengths. It will be shown that this difference in the level of electrotonic coupling leads to different degrees of coordination of burst activity between cells located on the same (cells strongly coupled) and opposite sides of the buccal mass (cells weakly coupled).

Part of the results of this paper have been published in abstract form (Egelhaaf, $1981 b)$.

\section{MATERIALS AND METHODS}

Specimens of Lymnaea stagnalis, obtained from Gerrard and Haig Ltd., East Sussex, were kept in aerated tap water and fed on lettuce. Experiments were carried out at room temperature in Hepes-buffered saline of the following composition (тм): $\mathrm{Na}^{+}, 24 \cdot 0 ; \mathrm{K}^{+}, 2 \cdot 0 ; \mathrm{Ca}^{2+} ; 4 \cdot 0 ; \mathrm{Mg}^{2+}, 2 \cdot 0 ; \mathrm{Cl}^{-}, 38 \cdot 0 ; \mathrm{H}_{2} \mathrm{PO}_{4}^{-}, 0 \cdot 1 ; \mathrm{Na}-\mathrm{Hepes}$ (Sigma), 35.4. Glucose $(15 \mathrm{mg} / \mathrm{l})$ was added to this solution. $\mathrm{A}$ high $\mathrm{Mg}^{2+} /$ zero $\mathrm{Ca}^{2+}$-saline was used to test for chemical synaptic junctions in a few experiments. Its composition was as follows (mM): $\mathrm{Na}^{+}, 55 \cdot 0 ; \mathrm{K}^{+}, 2 \cdot 0 ; \mathrm{Mg}^{2+}, 50 \cdot 0 ; \mathrm{Cl}^{-}, 142 \cdot 0$; $\mathrm{HCO}_{3}{ }^{-}, 15 \cdot 0 ; \mathrm{H}_{2} \mathrm{PO}_{4}{ }^{-}, 0 \cdot 1$; glucose, $15 \mathrm{mg} / 1$.

The buccal mass was dissected out under saline. The posterior jugalis muscle, which forms a thin sheath covering the posterior part of the buccal mass (Carriker, 1946; Egelhaaf, 1981a), was carefully removed to expose the underlying branches of the postbuccal nerve with the Peripheral Bursters attached to them (Fig. 1). Preparations were pinned to a layer of Sylgard in the bottom of a saline-filled watchglass. The buccal mass was slightly turned out of its natural position so that its posterior side 


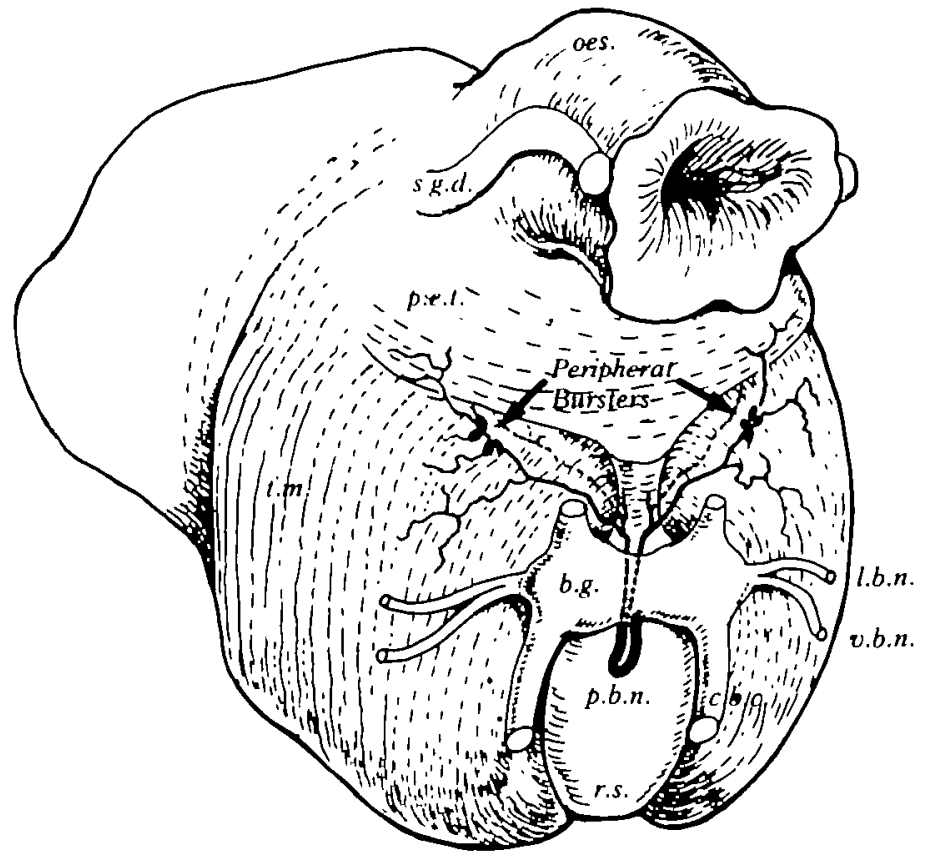

B

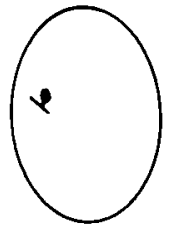

$\mathrm{C}$

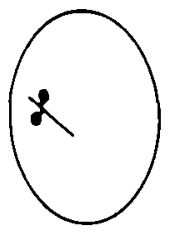

D

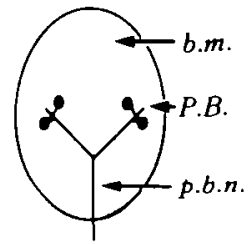

Fig. 1. (A) Location of Peripheral Bursters on the posterior side of the buccal mass. The oesophagus, salivary gland ducts and all buccal nerves, except the postbuccal nerve, have been removed. The posterior jugalis muscle has also been removed to expose the underlying network of Peripheral Bursters. (B)-(D) Schematic illustration of the different preparatıons used in this atudy. (B) Isolated cell preparation. (C) Network of Peripheral Bursters on one side of the buccal mass. (D) Entire network of Peripheral Bursters with only the CNS removed. Abbreviations: b.g. buccal ganglia; c.b.c. cerebrobuccal connective; oes. oesophagus; $l . b . n$. laterobuccal nerve; $P . B$. Peripheral Bursters; $p . b . n$. postbuccal nerve; $p$.e.t. pro-oesophagus tissue; $r . s$. radular sac; s.g.d. salivary gland duct; $t . m$. tensor muscle; v.b.n. ventrobuccal nerve; b.m. buccal mass.

faced upwards and the Peripheral Bursters were easily accessible. The dissection was completed in three different ways as described below.

\section{Isolated cells}

(Fig. 1B) All nerves which connect one Peripheral Burster with any other were cut. This difficult procedure was only feasible if the cell bodies were separated from each other by a sufficient gap. This preparation was used to demonstrate the endogenous bursting capability of Peripheral Bursters and to characterize qualitatively their intrinsic properties (Figs 3, 4C).

\section{Network of homolateral Peripheral Bursters}

(Fig. 1C) The cluster of Peripheral Bursters on one side of the buccal mass was isolated from the contralateral group by cutting both main branches of the postbuccal nerve distally to the side where it bifurcates. The superficial muscular layers were cut along the longitudinal midline of the posterior side of the buccal mass to prevent any interaction between heterolateral Peripheral Bursters via axonal branches which might not be detected under the dissection microscope. (The results shown in Figs 2, 4A, B, 5-8 were obtained using this preparation.) 


\section{Complete network of Peripheral Bursters}

(Fig. 1D) This preparation consists of the buccal mass with both left and right groups of Peripheral Bursters attached to it. This peripheral nerve net was separated from the CNS by cutting the postbuccal nerve just where it leaves the buccal ganglia as an unpaired nerve, while its main branches were still connected. This preparation was used to investigate the coupling of heterolateral Peripheral Bursters (Figs 9-13).

Intracellular recordings were made with glass microelectrodes filled with $0.5 \mathrm{M}$ $\mathrm{K}_{2} \mathrm{SO}_{4}$. Electrode resistances ranged from $50-80 \mathrm{M} \Omega$. The amplifiers used were equipped with a bridge balance system which allowed injection of current via the recording electrode. Injected current could be monitored by means of a currentvoltage converter situated in the indifferent line, between the saline in the recording dish and earth. Traces were displayed on a storage oscilloscope and permanently recorded, either on polaroid film or using a 2-channel pen recorder (Gould instruments). Pulsed or d.c. stimuli were provided by a Grass stimulator, connected via a stimulus isolation unit to the amplifiers.

\section{RESULTS}

The network of Peripheral Bursters consists of two clusters of 2-7 neurones which are located on the buccal mass quite separate from the ganglia of the CNS along bilateral branches of the postbuccal nerve (Fig. 1A). Their cell bodies, the diameter of which ranges between 10 and $40 \mu \mathrm{m}$, can be detected usually under the dissection microscope. In most cases they lie close together. Sometimes, however, they are somewhat distributed along the entire length of the nerve branch.

\section{Intrinsic properties of Peripheral Bursters}

The majority of Peripheral Bursters fire in spontaneously recurring bursts of spikes (Fig. 2A). Their burst frequency and number of spikes per burst fluctuate considerably. This is illustrated for a particular cell in Fig. 2B and C, where both period (Fig. 2B) and number of spikes per burst (Fig. 2C) are shown to be unimodally distributed. In the present section it will be established that bursting is an intrinsic property of Peripheral Bursters. It will be shown that the burst frequency is independent of tonically applied current and that application of short hyperpolarizing pulses can phase-shift the activity pattern, but only if they are applied during the burst and reduce the number of spikes.

\section{Bursting is an endogenous property of Peripheral Bursters}

The only way to demonstrate unambiguously the endogenous bursting capability of a neurone is to isolate the cell and thus separate it from any synaptic input. Mechanical isolation of cell bodies has been successively employed in a number of molluscan preparations (Alving, 1968; Chen, Baumgarten \& Takeda, 1971; Kostenko, Geletyuk \& Veprintsev, 1974; London \& Merickel, 1979). In the present study, in a few preparations (4) the Peripheral Bursters were sufficiently far apart to be isolated from the other Peripheral Bursters and the CNS by sectioning the nerve on either side of 

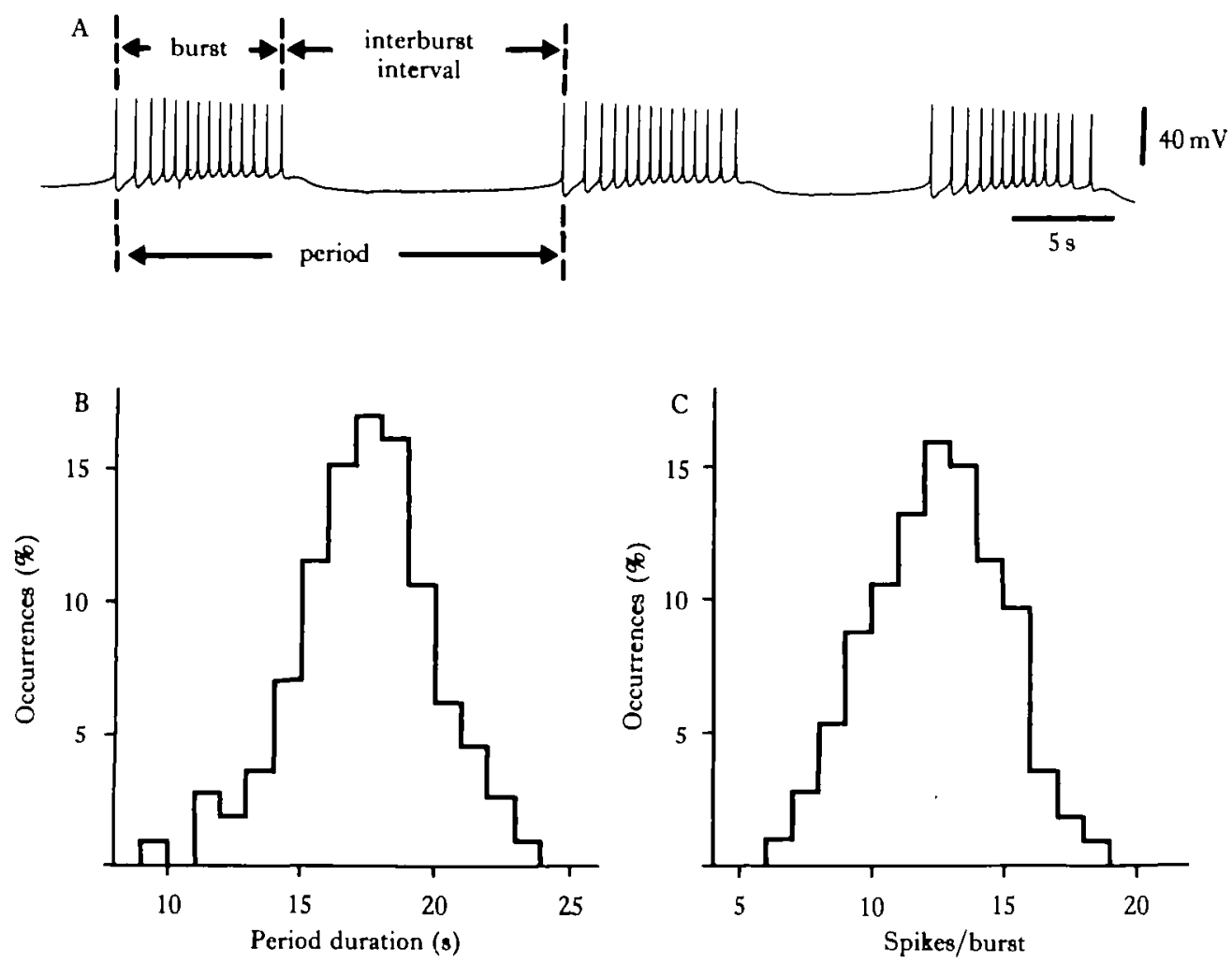

Fig. 2. Spontaneous activity of a Peripheral Burster showing the variability in the period and spikes per burst over a large number of cycles. (A) Sample record illustrating burst activity. (B) Perıod duration histogram of 113 cycles from a single cell. (C) Histogram of the number of spikes per burst of the same example as in (B). (Preparation as shown in Fig. 1C).

the cell body. In these preparations the bursting activity always persisted (Fig. 3A). Further evidence for the endogenous origin of bursting is given by two other tests (Fig. 3B, C). One test involved maintained hyperpolarization of an isolated cell to look for the presence of synaptic inputs; in the other, hyperpolarizing pulses were used to try to alter the bursting rhythm.

(i) Slight hyperpolarization of an isolated Peripheral Burster with a steady current occasionally revealed slow depolarizing waves without superimposed spikes (arrowed in Fig. 3B), occurring at the same frequency as the spontaneous rhythm. Judged by their wave form, these membrane potential waves are neither superimposed upon nor do they seem to be triggered by synaptic input, which can easily be identified in nonisolated cells (compare Fig. 3B with Fig. 8C). Thus bursting appears to be an endogenous cellular rather than a network property.

(ii) A hyperpolarizing current pulse applied during the burst, sufficiently large to terminate the spikes, shortens the following interburst interval, as is shown in another isolated cell preparation (Fig. 3C). The latter effect is more pronounced the earlier in the burst the hyperpolarizing pulse is applied. This phase-advancing effect of hyperpolarizing current pulses gives further evidence that bursting of Peripheral 
Bursters is an endogenous cellular property, since if it were driven by synaptic input the hyperpolarizing current pulses should not interfere with the rhythm (Selverston, 1980).

\section{Response to different levels of steady membrane polarization}

An interesting difference between Peripheral Bursters and other examples of endogenous oscillators is that their burst frequency does not depend on the level of membrane polarization. Fig. 4A shows the outcome of an experiment in which a steady transmembrane current of varying intensity was injected into a cell. The plot of average period duration versus applied transmembrane current (circles in Fig. 4A) reveals that the period duration of Peripheral Bursters is not affected by changes in transmembrane current. This is because the increase in burst duration with increasing depolarization (triangles in Fig. 4A) is associated with a corresponding decrease in

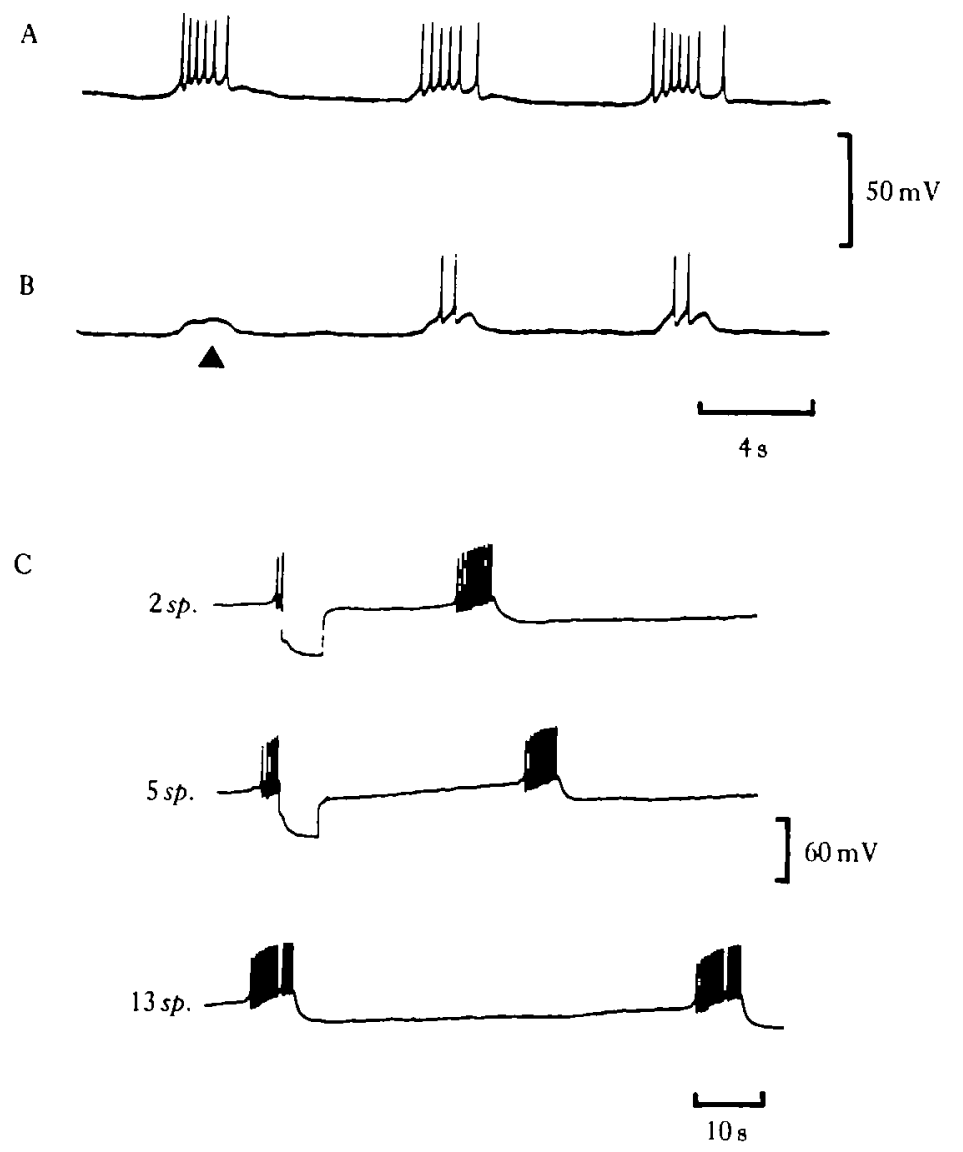

Fig. 3. Endogenous bursting capability of isolated Peripheral Bursters. (A) Spontaneous bursts in an isolated cell. (B) The same cell slightly hyperpolarized to prevent it from spiking. A slow membrane potential wave (see arrow) can be seen which is not surmounted by EPSPs. (C) Records from other isolated cell. The bursting thythm can be phase-advanced by hyperpolarizing current pulses injected during the burst. This effect depends on the number of spikes remaining in the burst shown on the left of each trace. No hyperpolarizing current pulse was applied in the bottom trace (sp., spikes). (Preparations as shown in Fig. 1B.) 
The average duration of the interburst interval (squares in Fig. 4A). With increasing burst duration the number of spikes per burst also increases (Fig. 4B, squares). However, since the number of spikes per burst increases to a greater extent than does the burst duration, the average spike frequency within a burst increases, too (Fig. 4B, circles).

The independence of burst frequency from the level of steady membrane polarization is probably an endogenous property of Peripheral Bursters, because it also occurs in cells isolated from other neurones. Evidence for this is given in Fig. $4 \mathrm{C}$, which
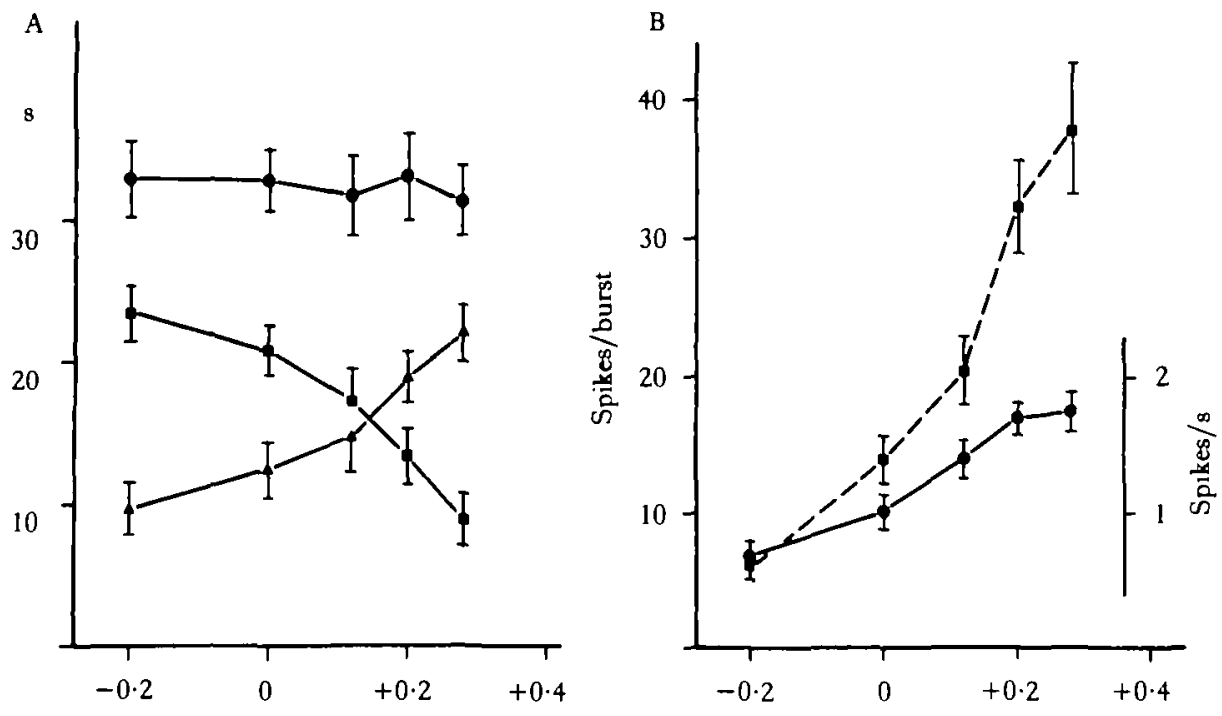

Injected current ( $\mathrm{n} A)$

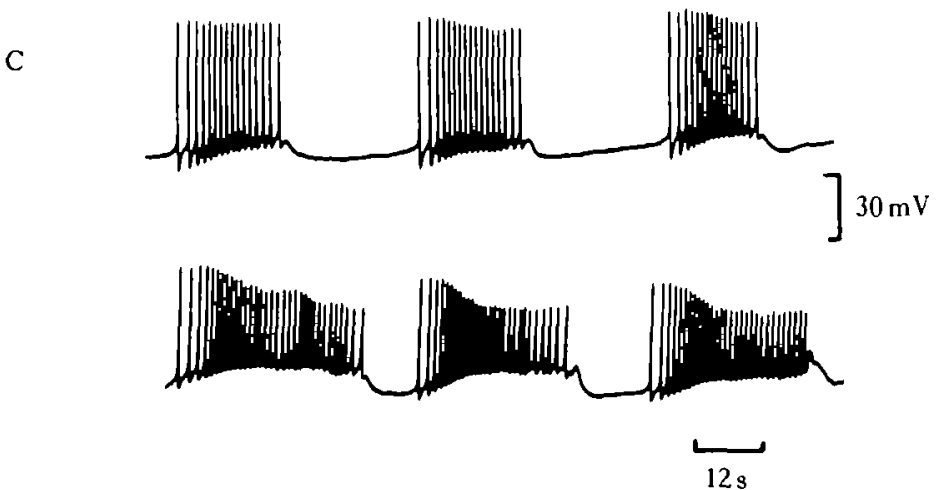

Fig. 4. Effects of changes in injected transmembrane current on the activity of Peripheral Bursters. (A) Average period duration (filled circles), interburst interval duration (squares) and burst duration (triangles) are plotted against the intensity of intracellularly injected current. Increasing levels of depolarization do not affect the average period duration due to the reciprocal relationship between interburst interval and burst duration. (B) The average number of spikes per burst (squares) and the average spike frequency (filled circles) are plotted against the intensity of injected current. Each point represents 10 cycles, except the points at $-0 \cdot 2 \mathrm{nA}$ which represent only 8 cycles. (Preparation used is shown in Fig. 1C.) (C) Records of burst activity in an isolated cell. Upper trace: no current is injected. Lower trace: depolarizing current is injected. (Preparation as shown in Fig. 1B.) 
shows sample records of the same isolated cell at two different levels of membrand polarization. In the upper trace no current has been injected, whereas in the lower trace a steady depolarizing current has been applied. Just as in cells in the intact homolateral network (Fig. 4A, B), the burst duration in this isolated cell increases following a depolarizing current injection. The interburst interval concomitantly decreases, thus leaving the cycle length unaltered.

\section{Perturbation of the bursting oscillator by short current pulses}

The rhythm of neuronal oscillators can be delayed or advanced in relation to the projected unperturbed rhythm by phasic input, such as synaptic potentials or short current pulses (Strumwasser, 1967; Pinsker, 1977a; Benjamin, 1978; Benson, 1980). These phase shifts depend on the phase of the bursting cycle at stimulus application. In the present study short $(0.5$ or $1.0 \mathrm{~s})$ hyperpolarizing current pulses have been applied to perturb the rhythm of Peripheral Bursters with a level of current intensity

A

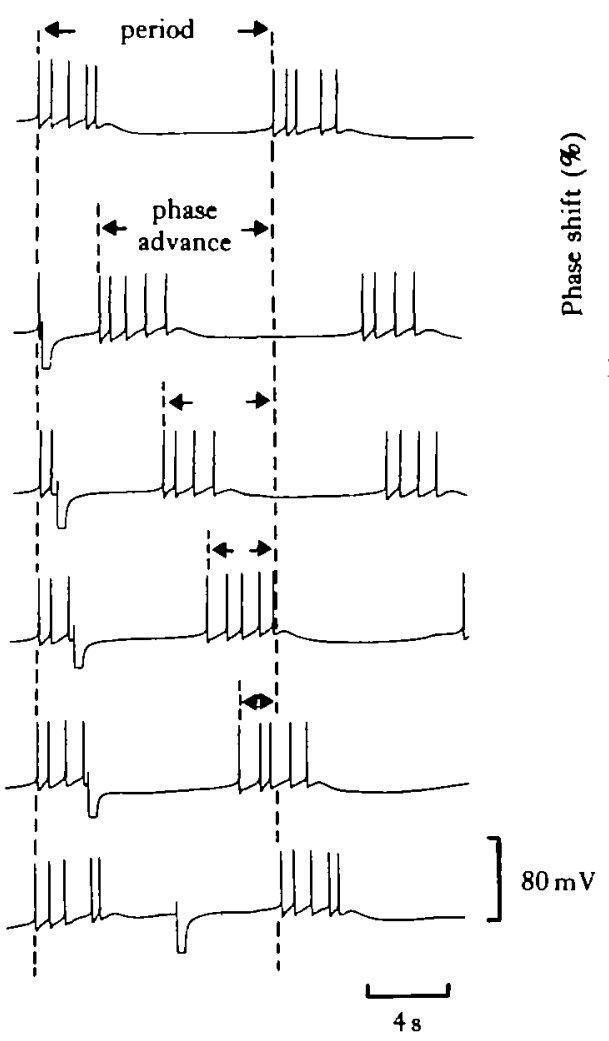

B

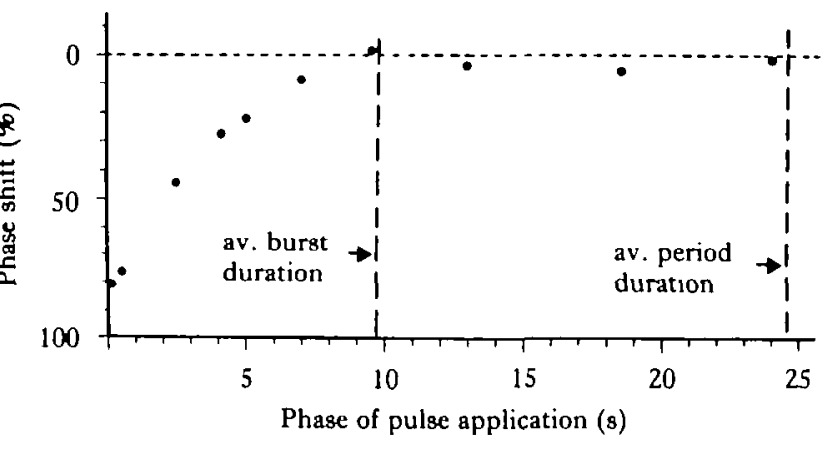

C

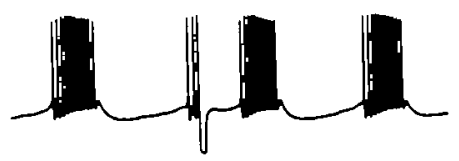

$40 \mathrm{mV}$

Fig. 5. Phase shiftıng effect of hyperpolarizing current pulses. (A) Sample records showing the effects of perturbing the oscillator at five different phases of the cycle by $0.5 \mathrm{~s}$ hyperpolarizing constant current pulses. The top trace shows an unperturbed cycle. Phase shift occurs only if the number of spikes in the burst is reduced by the stimulus. (B) Complete phase response curve showing the phase shift (in $\%$ of the average duration of unperturbed cycles) plotted against the phase of perturbation (in s). The average burst and period durations are indicated by vertical dashed lines. (C) Two sample records showing that the phase advancing effect of hyperpolarizing stimuli is independent of their duration. (Preparations as shown in Fig. 1C.) 
ist sufficient to terminate spiking, when the pulse was applied during the burst. Fig. 5 shows that in contrast to other molluscan bursting neurones (Pinsker, 1977a; Benjamin, 1978) only phase advance can be induced with hyperpolarizing stimuli (for definition see Fig. 5A).

The effect of hyperpolarizing current pulses applied at different phases of the bursting cycle is illustrated in the recordings of Fig. $5 \mathrm{~A}$. When the hyperpolarizing pulse is presented just after the first spike in a burst, the burst is terminated and the occurrence of the next burst is advanced to some extent. The phase advance decreases as the hyperpolarizing pulse is given later in the bursting cycle. If the pulse is applied anywhere during the interburst interval, neither phase advance nor phase delay occurs. Since the durations of the periods following the perturbed cycle are always within the range of spontaneous variation, there are no enduring changes in cycle length and the full phase shift is expressed within one cycle.

The phase shift can be presented as a phase response curve which is defined as the plot of the phase shift as a percentage of the average duration of unperturbed cycles versus the phase of the bursting cycle at which the stimulus has been applied (Pavlidis, 1973). The phase response curve in Fig. 5B again illustrates that the phase advance decreases gradually and finally leads to a zero phase shift, as the hyperpolarizing pulse is applied successively later in the bursting cycle. The duration of the stimulus does not influence the phase shift, as the sample records in Fig. $5 \mathrm{C}$ illustrate.

\section{Phase response curves at different levels of membrane polarization}

In Peripheral Bursters the shape of the phase response curve following application of hyperpolarizing current pulses depends on the level of membrane polarization.

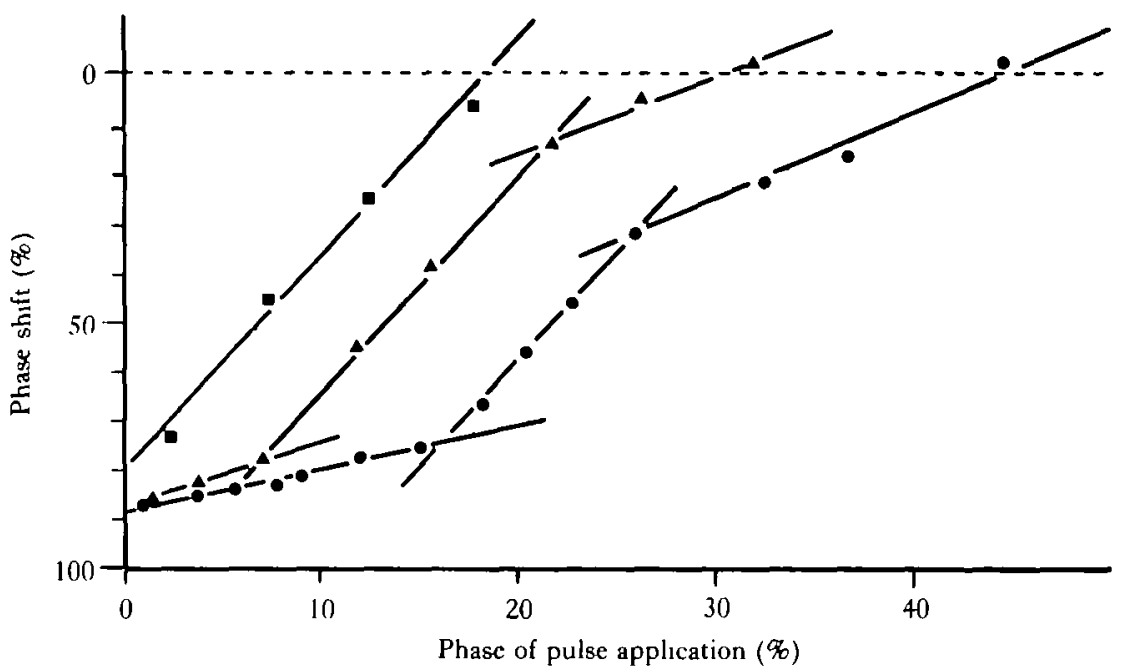

Fig. 6. Effects of different levels of membrane polarization on the phase shift elicited by $0.5 \mathrm{~s}$ hyperpolarizing current pulses. Triangles, no steady current injected; squares, hyperpolarizing current injected; circles, depolarizing current injected. Since hyperpolarizing current pulses injected during the interburst interval have no phase shifting effect (see Fig. 5) they were only given during the burst. Phase shift (in $\%$ of the average duration of unperturbed cycles) is plotted against the phase of stumulus application (in $\%$ of the average unperturbed cycle length). (Preparation as ahown in Fig. 1C.) 
This is different from the only other example known to us of an endogenous oscillatc in which this has been studied in detail (Pinsker, 1977a). Phase response curves havē been obtained for the same cell at different levels of tonically applied trans-membrane current. Since hyperpolarizing current pulses, injected during the interburst interval, have no phase shifting effect (Fig. 5), they were given only during the burst.

As previously explained, the phase advance depends on the phase of the cycle at which the pulse is applied and this is true for all levels of membrane polarization shown in Fig. 6. However, the shape of the phase response curve is altered as a result of changes in sign and intensity of tonically injected current. If the cell is hyperpolarized, the phase response curve can be represented by a straight line (squares in Fig. 6). In the non-polarized or depolarized cell it is sigmoid and, in the first approximation, three subsequent straight line segments can be fitted to the data (triangles and circles in Fig. 6). The shallow-slope parts at the beginning and end of the phase response curve are relatively short in the non-polarized cell; they become longer if depolarizing current is injected.

\section{Relation between spike number and interburst interval at different levels of membrane polarization}

As was indicated in Fig. 5, phase advance induced by short hyperpolarizing current pulses is due to a reduced number of spikes in the preceding burst. This suggests that the number of spikes in a burst is an important determinant of the next interburst interval and this is examined in detail in the present section. At three levels of tonically injected transmembrane current the relationship between interburst interval and the number of spikes in the preceding burst was measured for bursts truncated by applying hyperpolarizing pulses as well as for unperturbed cycles. The latter has been possible because of the variability in the number of spikes in spontaneously generated bursts (Fig. 2C). The following interpretations emerge from these interburst interval curves:

(i) For artificially truncated bursts (filled symbols in Fig. 7), the dependence of the interburst interval curve on the level of membrane polarization is similar to that of the phase response curve (Fig. 6). Thus, for hyperpolarized cells the interburst interval curve is linear (squares in Fig. 7), whereas it becomes sigmoid when the cell becomes more depolarized (triangles and circles in Fig. 7). This shallowness at the beginning and end of the curve suggests that the spikes at the beginning and towards the end of the burst contribute only a small increment to the entire interburst interval compared with those in the middle part of the burst.

(ii) For unperturbed cycles, the relationship between interburst interval and the number of spikes in the preceding burst is linear (open symbols in Fig. 7); the slope of the regression line decreases as the cell becomes more depolarized (open circles) or conversely it becomes steeper as the cell is more hyperpolarized (open squares) compared with the unpolarized situation (open triangles). This means that there is a smaller increment in the interburst interval for each additionally generated spike in the more depolarized state of the cells.

(iii) For a given level of membrane polarization, the duration of interburst intervals succeeding truncated bursts with a particular number of spikes (filled symbols in 


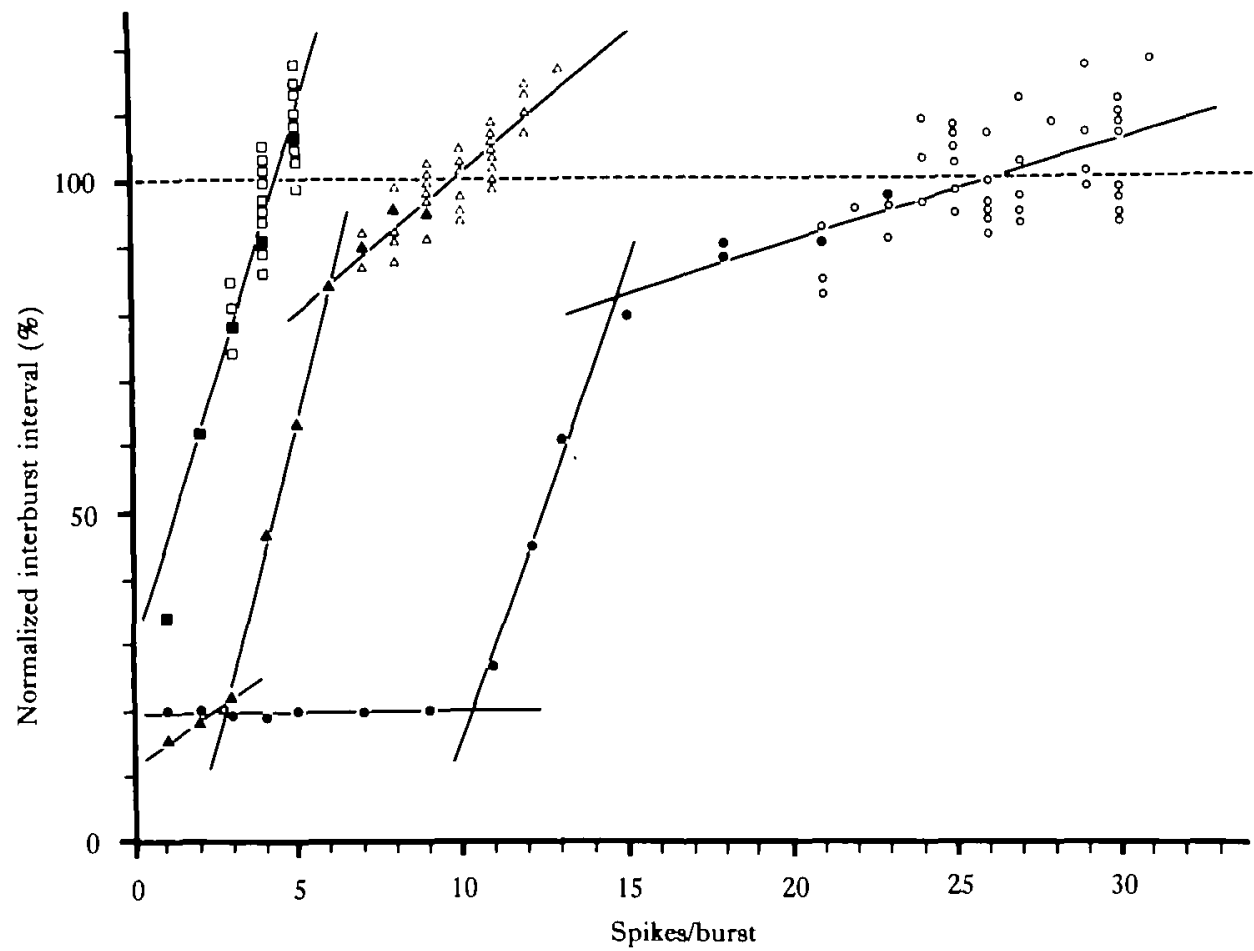

Fig. 7. Effects of different levels of membrane polarization on the interburst interval. The shape of the interburgt interval curve is influenced by the level of injected current, but at a given level the interburst interval depends only on the number of spikes in the preceding burst regardless of whether or not it is truncated by a short hyperpolarizing pulse. Triangles, no current injected; circles, depolarızing current injected; squares, hyperpolarizing current injected. Open symbols represent unperturbed bursts; filled symbols represent bursts truncated by $0.5 \mathrm{~s}$ hyperpolarizing current pulses.

The curves have been approximated by regression lines. (Same example as in Fig. 6.)

Fig. 7) is always within the range of interburst intervals following unperturbed bursts with the same number of spikes (open symbols in Fig. 7). This gives some evidence that the perturbation of the bursting oscillator by hyperpolarizing current pulses only prevents spikes from occurring, which alone leads to a shortened inter-burst interval; it does not affect the oscillator in any other obvious way.

\section{Network interactions among Peripheral Bursters}

The system of Peripheral Bursters offers an excellent opportunity for examining the effect of different levels of coupling on burst coordination, because homolateral and heterolateral Peripheral .Bursters form two distinct cell populations with respect to their coupling strength. As will be shown below, homolateral cells are relatively strongly coupled, showing a high degree of correspondence in their burst and spike activity, whereas heterolateral cells are only weakly coupled, as is reflected in a higher degree of variability in their patterns of coordinated burst activity.

\section{Interactions among homolateral Peripheral Bursters}

Peripheral Bursters on the same side of the buccal mass always burst together and in most cases spike synchrony within bursts also occurs, as is shown by recording 
A
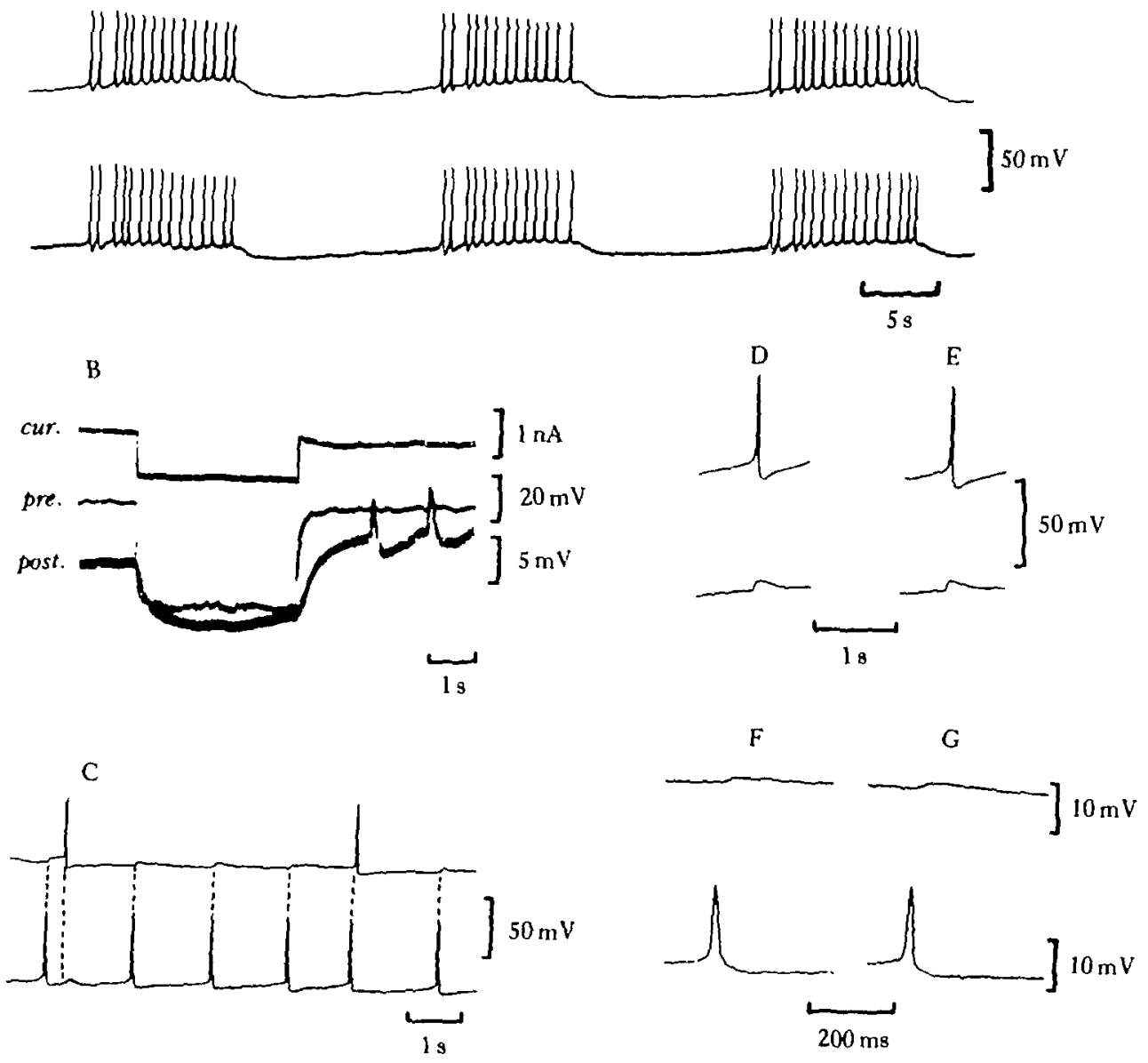

Fig. 8. Network interactions of Peripheral Bursters on the same sude of the buccal mass. Simultaneous recordings from two cells. (A) Burst and spike synchrony. (B) Cells on the same side are electrotonically coupled; an intracellularly injected current pulse (cur.) leads to a membrane potential shift in the cell into which it has been injected (pre.) as well as in another ipsilateral cell (post.). (C) EPSPs in a slightly hyperpolarized cell (upper trace) occur simultaneously with spikes in another ipsilateral cell (lower trace). (D), (E) Transmission is unaffected by alteration of the postsynaptic membrane potential: (E) hyperpolarizing the pastsynaptic cell by approximately $20 \mathrm{mV}$ (lower trace) does not affect the EPSP amplitude. For comparison the same cell is not hyperpolarized in the lowes trace in (D). (F), (G) High $\mathrm{Mg}^{2+} /$ zero $\mathrm{Ca}^{2+}$-saline does not affect coupling. (F) Preparation in Hepes-saline. (G) Preparation in high $\mathrm{Mg}^{2+} /$ zero $\mathrm{Ca}^{2+}-8 a l ı n e$ for approximately $20 \mathrm{~min}$. (Preparations as shown in Fig. 1C.)

simultaneously from two cells (Fig. 8A). This synchrony is due to relatively strong electrotonic coupling of homolateral cells rather than to interactions by chemical synapses. The evidence for electrotonic coupling is as follows. A constant current step injected into a cell is always accompanied by a membrane potential shift of corresponding sign and duration in another ipsilateral cell, as is illustrated in Fig. 8B for a hyperpolarizing pulse. To ensure that the response was not an artifact, the injection electrode was withdrawn from the cell and no response was now seen. The d.c. coupling coefficient, measured as the ratio of membrane potential change in the postsynaptic cell to that of the presynaptic cell (Bennett, 1977) varies between 0.15 
And 0.45 in different preparations. As a consequence of the low-pass filter characteristics typical for electrotonically coupled cells (Getting, 1974; Merickel, Eyman \& Kater, 1977), the coupling coefficient of Peripheral Bursters was also shown to depend on the frequency of the presynaptic current signal (Egelhaaf, 1981a).

In a cell slightly hyperpolarized by injected current, unitary EPSPs are elicited by each spike in another ipsilateral cell which occasionally also evoke action potentials (Fig. 8C). This shows that electrotonic coupling can lead to 1:1 firing in homolateral Peripheral Bursters. If the postsynaptic cell was hyperpolarized by approximately $20 \mathrm{mV}$ (lower trace in Fig. 8E), there was no detectable change in the amplitude of the EPSPs (compare Fig. 8E with Fig. 8D), which is consistent with the EPSPs being electrotonically mediated rather than being due to chemical synaptic transmission. Moreover, transmission is not affected by high $\mathrm{Mg}^{2+} /$ zero $\mathrm{Ca}^{2+}$ saline (Fig. $8 \mathrm{~F}, \mathrm{G}$ ), which is reported to block chemical synapses (Nicholls \& Baylor, 1968).

\section{Interactions among heterolateral Peripheral Bursters}

While the group of homolateral cells was shown to burst synchronously due to relatively strong electrotonic coupling and can thus be regarded as a single oscillatory unit, the activity of heterolateral cells is less closely related (Fig. 9). Since this variability makes it difficult to illustrate the activity of heterolateral cells in short bursting sequences, two other kinds of data presentation will be given to include longer bursting sequences into the analysis: (i) The period duration as well as the time shift between the onset of corresponding cycles are plotted versus time (for definition of 'corresponding cycles' see legend of Fig. 9). (ii) The frequency of occurrence of variables such as period duration, time shift between corresponding cycles and the ratio of period durations of corresponding cycles are displayed in order to study the coordination of burst activity quantitatively.

The average burst frequencies of heterolateral cells deviate from each other to a different extent in all preparations from which recordings were made. This is illustrated qualitatively for three representative pairs of heterolateral cells from different preparations in the sequential period duration plots (upper diagrams in

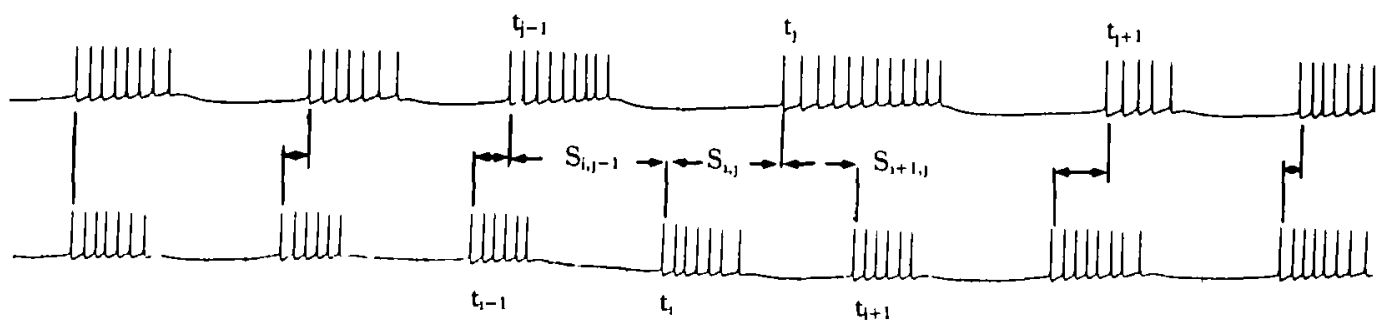

Fig. 9. Burst activity of heterolateral Peripheral Bursters showing the parameters measured for analysing burst relationships in subsequent figures. Simultaneous recording from two heterolateral cells. The discharge pattern of the cell with the lower average burst frequency (upper trace) will be used 28 the reference trace. The time of beginning of bursts in this cell will be denoted by ...t,-1, 4 , $t_{j+1} \ldots$ If a burst in the cell with the higher burst frequency (lower trace) begins at a time $t_{j-1}<t_{1}$ $<t_{1}$, the differences $S_{i, j-1}=t_{i}-t_{j-1}>O$ and $S_{i, j}=t_{i}-t_{j}<O$ are calculated and called time shifts. If $\left|S_{i, j}\right|<\left|S_{i, j-1}\right|\left(\left|S_{i, j-1}\right|<\left|S_{i, j}\right|\right)$, the cycle of the cell with the higher burst frequency starting at $t$, will be related to the cycle of the cell with the lower burst frequency starting at $t_{1}\left(t_{1}-1\right)$; these cycles will be called corresponding cycles. (Preparation as shown in Fig. 1D.) 

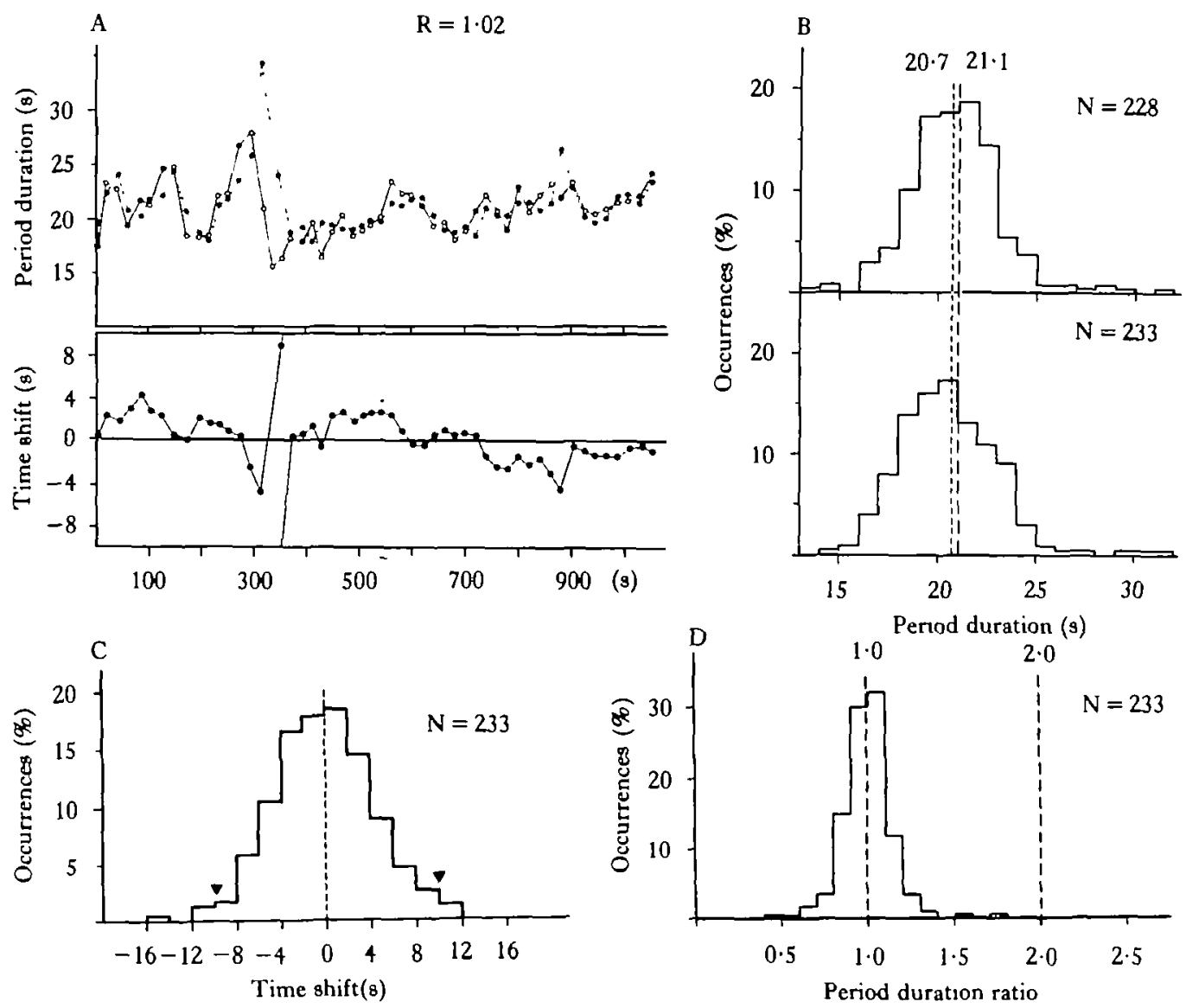

Fig, 10. Burst activity of heterolateral Peripheral Bursters. (A) The period duration of two heterolateral cells (upper diagram) and the time shift between corresponding cycles (lower diagram) are displayed versus time. $\mathrm{R}$ is the ratio of average burst frequencies of corresponding heterolateral cells. (B) Period duration histograms, (C) histogram of the time shift between corresponding cycles and (D) histogram of the period duration ratio of corresponding cycles of the same pair of cells as in (A). The histograms in (B)-(D) include more cycles than the time-dependent plot in (A). Arrowheads in (C) indicate time shifts of half an average cycle. (Preparation as shown in Fig. 1D.)

Figs 10A, 11A, 12A) and quantitatively in the corresponding period duration histograms (Figs 10B, 11B, 12B). The period durations of the different cells fluctuate considerably, but their distributions have distinct peaks. They are shifted with respect to each other in the different cell pairs and the ratio of their means $(R)$ can be used as a measure of displacement of the respective period duration histograms. In the example of Fig. 10B, the period duration distributions match each other almost completely and their respective means differ only slightly $(R=1.02)$. In Fig. 11B, the distributions are somewhat displaced and their means have a ratio of $R=1.20$, whereas in Fig. 12B both distributions overlap only a little and $R$ amounts to 1.64 . Hence, there is a wide range of possible period duration ratios between pairs of heterolateral cells in different preparations.

Despite this variability in the average period durations of coupled heterolateral cells their burst activity is in some way coordinated. This can be concluded even from the 

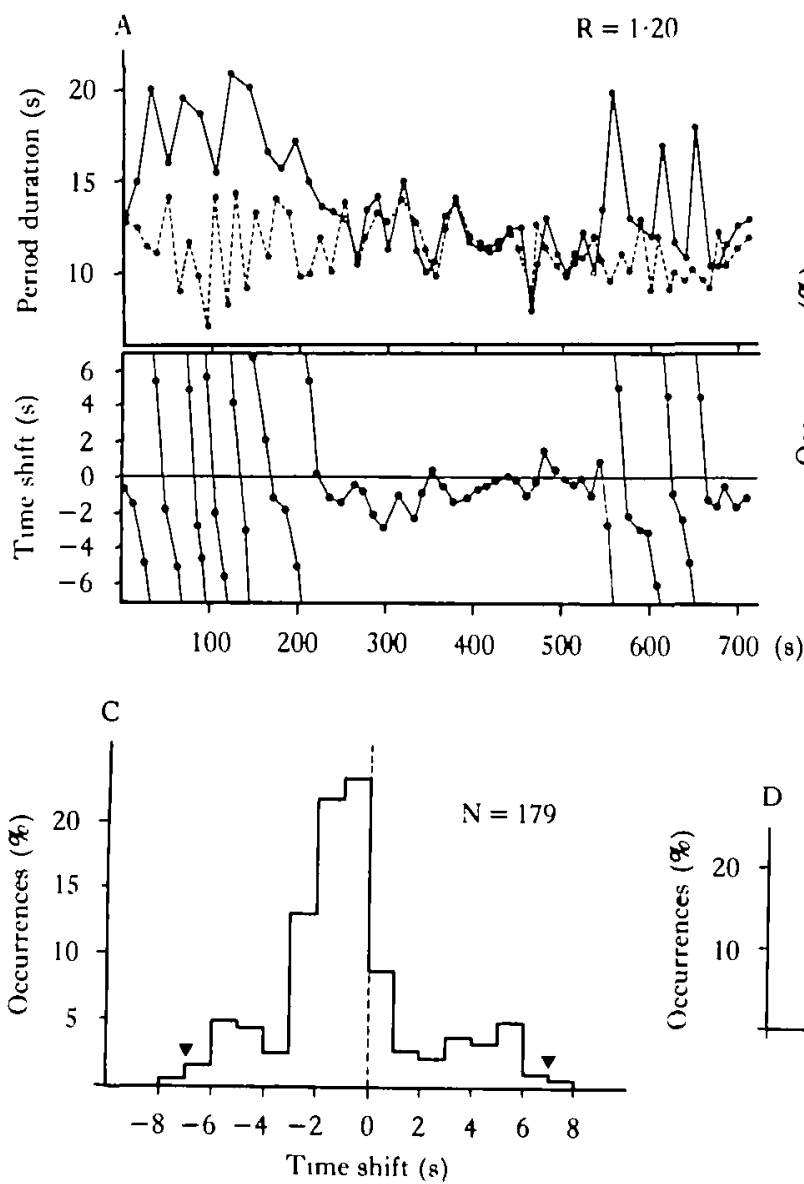

B

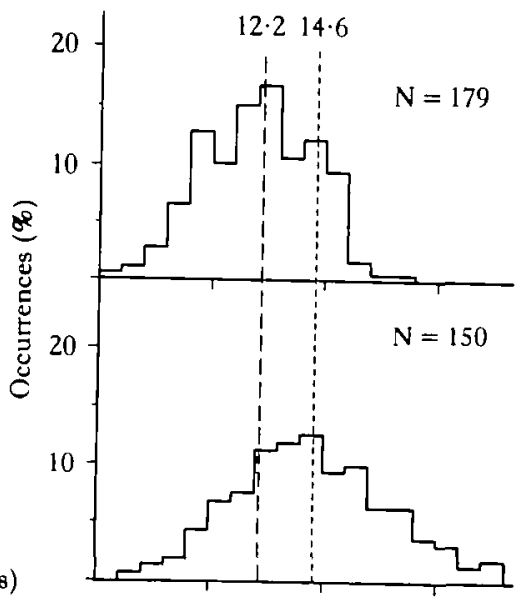

20

Period duration (s)

Fig. 11. Burst activity of another pair of heterolateral Peripheral Bursters. $R$ amounts to $1 \cdot 20$ in this example. (A)-(D) As in Fig. 10.

time based plots, since in all three examples shown in Figs 10-12 periods with $1: 1$ entrainment occur. This is reflected by nearly identical period durations in the coupled cells for at least some consecutive cycles during which fluctuations in period duration are usually closely matched. During these periods of $1: 1$ entrainment the time shift between corresponding bursts in the two cells is small in relation to the cycle length (lower diagrams of Figs 10A, 11A, 12A). However synchronous bursting is interrupted in all three examples.

Quantitative evidence for coordinated burst activity in heterolateral cells is provided in histograms showing the frequency of occurrence of the different time shifts between corresponding cycles. (Figs 10C, 11C, 12C). Since in all three examples certain time shifts occur more frequently than others, both rhythms are not running independently and some sort of coupling must exist. However, the shape of the time shift histograms depends on the difference in average period duration. In pairs of cells with almost identical average period durations it is bell-shaped (Fig. $10 \mathrm{C}$ ), whereas in the extreme case of an average period duration ratio of $R=1 \cdot 64$ the histogram has three distinct peaks, one for small time shifts and two for positive and 

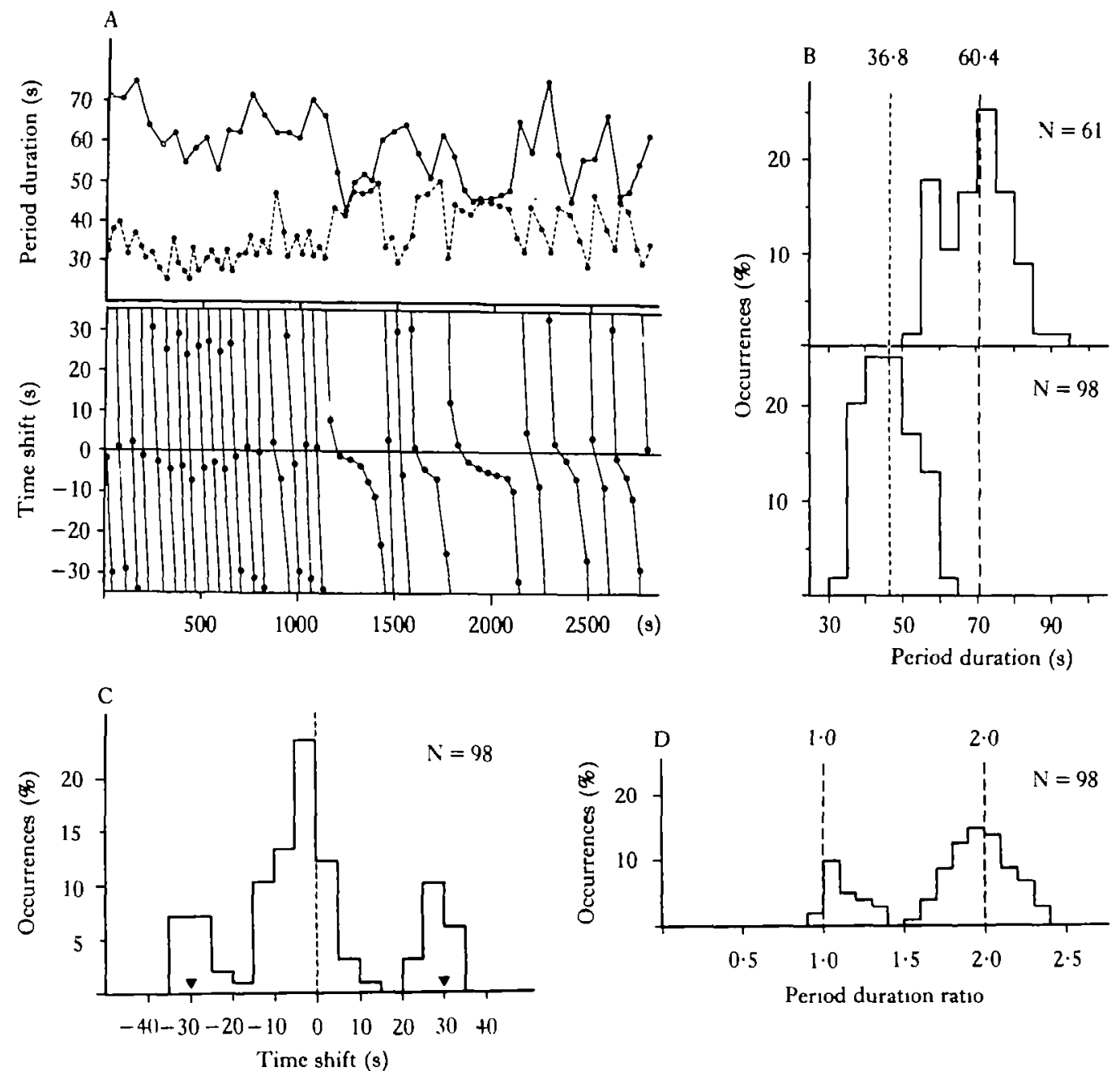

Fig. 12. Burst activity of another pair of heterolateral Peripheral Bursters. $R$ amounts to $1 \cdot 64$ in this example. (A)-(D) As in Fig. 10.

negative time shifts of about half a cycle (Fig. 12C). (Note that delays and advances of half a cycle are virtually the same!) The shape of the time shift histogram of the example with $R=1.20$ is intermediate between these extreme examples (Fig. 11C). Hence irrespective of the average period duration ratio small time shifts between corresponding bursts represent a preferred interoscillatory phase relationship. However, in cases with large differences in the average burst frequency additional preferred phase relations occur.

When periods of $1: 1$ entrainment are interrupted by periods without such entrainment, the ratio of burst frequencies is frequently close to $1: 2$. This can be inferred from the histograms of period duration ratios of corresponding cycles (Figs 10D, 11D, 12D) since these have peaks at 1.0 and $2 \cdot 0$, independent of the difference in average burst frequency. However, the frequency of occurrence of both values is different in the different preparations and depends on the difference in average burst frequencies 
f the coupled cells. In particular, in the example of cells with almost completely verlapping period duration distributions there is only one peak at $1 \cdot 0$. Hence, the burst frequencies of heterolateral cells most commonly have the ratio $1: 1$ or $1: 2$.

This relatively weak synchronization is presumably due to a lower electrotonic coupling efficiency between heterolateral cells compared with homolateral ones. Evidence for this is given in Fig. 13A, where intracellularly injected current into a cell leads to only a small membrane potential shift of corresponding sign and duration in a heterolateral cell. No response occurred when the current injection electrode was removed from the cell. The d.c. coupling coefficient of approximately 0.05 obtained in this example was at the upper range of coupling coefficients found in heterolateral cells. In general, the coupling was always considerably lower compared with that of homolateral cells (compare Fig. 13A with Fig. 8B). In some preparations small EPSPs (approximately $0.2 \mathrm{mV}$ in Fig. 13B) can be seen following $1: 1$ spikes in a contralateral cell. In most preparations, however, it was impossible to detect EPSPs above the noise level.

Since intracellularly injected current signals propagate only passively, the coupling coefficient is not a good way of characterizing the real strength of coupling in examples where injection and recording sites are considerably apart (McCrohan \& Benjamin,

A
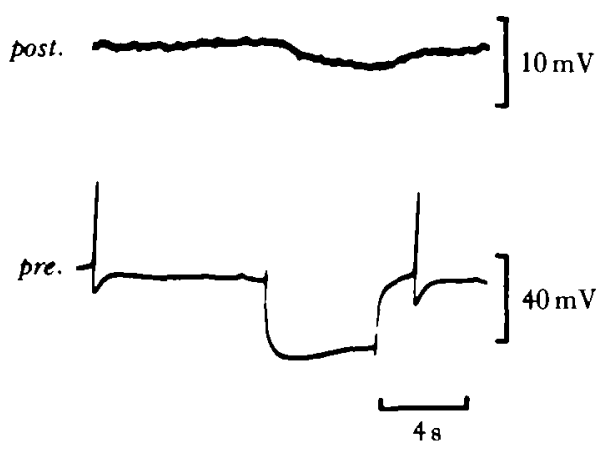

B

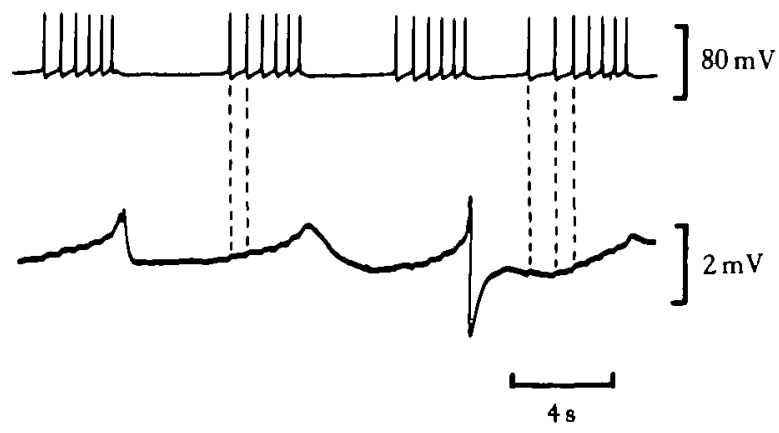

Fig. 13. Electrotonic coupling among heterolateral Peripheral Bursters. (A) An intracellularly injected current pulse leads to a membrane potential shift in the cell into which the pulse has been injected (pre.) and in a heterolateral cell (post.). (B) In records with a low noise level EPSPs can be seen following spikes in a heterolateral cell. (Preparation as shown in Fig. 1D.) 
1980). Therefore, it cannot be decided on the basis of such experiments, whether th relatively weak synchronization of heterolateral Peripheral Bursters is the result of less efficient electrotonic junctions compared with those connecting homolateral cells, or the result of signal attenuation due to a large distance between the coupling site and the region of the bursting pacemaker.

\section{DISCUSSION}

Since the network of Peripheral Bursters can be subdivided into different subsystems by cutting particular nerves connecting the cell bodies together (Fig. 1B-D), it provides a good model system for studying the contribution to pattern generation of both endogenous cellular properties and network interactions due to electrotonic coupling. Consequently the endogenous properties of Peripheral Bursters have been analysed, at least qualitatively, separately from the modulation of their activity by synaptic input. In particular, it was shown that the bursting capability of Peripheral Bursters is an intrinsic property of single cells (Fig. 3).

Although this could be demonstrated unambiguously in only a few preparations because of methodological difficulties, we think that all Peripheral Bursters are equivalent with respect to their functional properties. Further evidence for this is as follows. First, the global features of axonal geometry of all cells injected with Lucifer Yellow were found to be very similar (Egelhaaf, 1981a). Second, no principal electrophysiological differences between any Peripheral Bursters could be detected. Third, the variable number of Peripheral Bursters in different preparations, as well as on opposite sides of the buccal mass in the same preparation, is an indication that no fixed number of them is necessary to accomplish their function, whatever it might be, and that no specific functional role can be attributed to a particular cell within the network.

\section{Intrinsic properties of Peripheral Bursters}

\section{Peripheral Bursters as neuronal oscillators}

In studies designed to characterize a bursting oscillator, by variables such as spike numbers and duration of burst and interburst interval, the bursting cycles are perturbed at different phases either by controllable synaptic input (Pinsker, 1977a,b; Ayers \& Selverston, 1979) or, as in the present study, by short current pulses (Strumwasser, 1967; Mayeri, 1973a,b; Pinsker, 1977a; Benson, 1980) and the induced phase shift or the effect on the interburst interval studied. In Peripheral Bursters, as in these other examples, the induced phase shift depends on the number of spikes already generated when the burst terminating stimulus is applied. In Peripheral Bursters only phase advance can be induced by hyperpolarizing current pulses, and this effect decreases towards the end of the burst. No phase shift is induced as the pulse is applied during the interburst interval (Fig. 5). In this respect Peripheral Bursters are similar to the cardiac ganglion of Portunus (Benson, 1980), the PD-unit in the stomatogastric ganglion of the lobster (Ayers \& Selverston, 1979) and, if short duration hyperpolarizing pulses were used to perturb the rhythm, to the LUQ-bursters of Aplysia (Pinsker, 1977a). In contrast, stimulation during the interburst interval delays the onset 
ff the next burst in Yellow Cells of Lymnaea using hyperpolarizing current pulses Benjamin, 1978) and in the LUQ-bursters of Aplysia when long current pulses or synaptic stimuli were applied (Pinsker, 1977a). In Peripheral Bursters it was shown that the induced phase shift is independent of the duration of the current pulses, if the latter do not extend into the next burst (Fig. 5C).

The difference in the phase response curves between Lymnaea Peripheral Bursters and Aplysia LUQ-bursters might be due to the fact that apart from altering the number of spikes per burst and, concomitantly, the interburst interval duration, perturbing stimuli can affect the oscillator directly in LUQ-bursters, whereas they do not in Peripheral Bursters. Evidence for this is as follows. In LUQ-bursters the phase shift induced by synaptic stimulation or 'long' current pulses consists of two components, an advancing component which is inversely proportional to the number of remaining spikes and a phase delaying component which reflects a direct interaction between stimulus and the pacemaker oscillations (Pinsker, 1977a). In contrast, in Peripheral Bursters only a phase advancing effect, which is directly related to the number of spikes remaining in the burst, can be ascribed to the hyperpolarizing current pulses, since for a given number of spikes per burst the following interburst interval in perturbed and unperturbed cycles does not differ significantly (Fig. 7).

\section{Response of Peripheral Bursters to different levels of membrane polarization}

In Peripheral Bursters of Lymnaea, burst frequency does not change significantly following changes in membrane polarization (Fig. 4). In all other examples of similar experiments known to us (e.g. Arvanitaki \& Chalazonitis, 1967; Selverston et al. 1976; Pinsker \& Kandel, 1977; Benson, 1980), the burst frequency increases following a decrease in membrane potential. This different influence of tonically-applied transmembrane current on burst frequency is reflected in the different shape of the phase response curves in Peripheral Bursters of Lymnaea and the LUQ-bursters of Aplysia (Pinsker, 1977a), the only other example in which phase response curves were obtained at different levels of membrane polarization.

In the LUQ-bursters in Aplysia the shape of the phase response curve remains unaltered, when the membrane potential is changed, and it can be concluded from Pinsker's (1977a) data that the contribution of spikes to the interburst interval is independent of the level of membrane polarization. As a result the period duration decreases following depolarization of the cell, since then the number of spikes per burst decreases, too (Pinsker, 1977a). Similarly Benson (1980) obtained a linear relationship between the duration of spontaneously generated bursts and the succeeding interburst interval in the cardiac ganglion of Portunus, irrespective of the level of membrane polarization at which the burst was generated.

In contrast, in Peripheral Bursters both phase response and interburst interval curves are affected by polarization of the cell. The curves are linear (for pulses applied during the burst) when the cell is tonically hyperpolarized, but are transformed to a sigmoid function, as the cell becomes more depolarized (Figs 6, 7). As a result, only a small number of spikes contribute the major increment to the phase shift and interburst interval duration, and these are associated with the steep part of the curves. Additional spikes in bursts in a more depolarized cell contribute only little to the phase 
shift and interburst interval duration, leaving the period duration unaffected by changes in membrane polarization.

\section{Network interactions among Peripheral Bursters}

\section{Coordination of burst activity as a function of coupling strength}

Two or more oscillators can be coupled in such a way that they mutually entrain or interact with each other. On the cellular level there are many studies on electrotonically coupled neurones (for review see Bennett, 1977) but only a few are directly concerned with the coordination of the cell's oscillatory output as a function of coupling strength. For instance, there are studies on the coupling strength dependent mutual entrainment of spike activity in embryonic heart cells (Ypey, Clapham \& DeHaan, 1979). However, dealing with the coordination of bursting neurones by electrotonic coupling, with one exception (van Swigchem, 1981), only results from strongly coupled bursting cells (e.g. Kaneko, Merickel \& Kater, 1978; Tazaki \& Cooke, 1979) have been previously reported. Therefore, it was interesting to be able to study, within a given neuronal network, how the coordination of burst activity of different equivalent autoactive pattern generators depends on their coupling strength. Another network property of Peripheral Bursters, the restoration of spike synchrony within bursts of homolateral cells which tend to fire asynchronously, has been discussed elsewhere (Egelhaaf, 1981a,b; Egelhaaf \& Benjamin, 1982).

Both homo- as well as heterolateral Peripheral Bursters are electrotonically coupled, although with different coupling strength. Coupling of homolateral cells is strong enough always to produce burst synchrony. In contrast, heterolateral cells are only weakly coupled (compare Fig. 8 with Fig. 13). This allows them to burst at different average frequencies, and their patterns of coordinated burst activity are more variable (Figs 10-12). In fact they show most commonly a succession of periods with $1: 1$ and $1: 2$ entrainment. Which of the two entrainment modes prevails, differs in different preparations (Figs 10-12).

Phenomenologically similar coupling strength-dependent features of coordination of neuronal oscillators have been obtained in a computer analysis of coupled model pacemaker cells with different intrinsic frequencies (Ypey, Van Meerwijk, Ince \& Groos, 1980). Here, stable 1:1 entrainment occurred, when the coupling resistance was low, but the mode of entrainment changed when the coupling resistance was increased. Around certain values of coupling strength stable 1:2 entrainment occurred. Nevertheless, the model simulation of Ypey et al. (1980) differs in one important respect from the experimental results obtained in Peripheral Bursters, since in the latter case within single preparations, i.e. for a given coupling strength, both $1: 1$ and $1: 2$ entrainment and transitions between these two entrainment modes occur (Figs 11, 12). This difference might be accounted for by the stochastic intrinsic burst frequency behaviour of Peripheral Bursters (Fig. 2B), which is different from the model simulation of Ypey et al. (1980), where the coupled pacemaker cells had given fixed intrinsic frequencies.

Studying a more complex synaptic relationship among bursting model neurones by computer simulation, Mulloney, Perkel \& Budelli (1981) came to conclusions quite in accordance with our experimental findings. Although their model neurones were 
noupled by both electrotonic junctions as well as chemical inhibitory synapses, they lound that (for a given strength of inhibitory coupling) an increase in electrotonic coupling strength tended to synchronize bursting.

In conclusion, Peripheral Bursters mutually entrain each other as a consequence of their electrotonic coupling. The degree of coordination which can be achieved in both homo- and heterolateral cells is directly related to the strength of their mutual coupling. In addition, the burst activity of Peripheral Bursters cannot only be mutually entrained within this peripheral network, but there are indications reported elsewhere (Egelhaaf, 1981 $a, b$ ) that it can be also entrained by the central nervous feeding pattern generator in preparations where the network of Peripheral Bursters remains connected to the CNS via the postbuccal nerve, which allows the buccal mass to perform cyclic feeding movements. Hence, the activity of Peripheral Bursters can be entrained not only by their own interactions but also by other outside elements.

M.E. is most grateful to Prof. D. Varjú for encouragement and help throughout this study. Prof. D. Varjú and H. Wagner are thanked for critical comments on previous drafts of this paper and Joanna Harper for typing the manuscript. M.E. was supported by a grant from the Studienstiftung des deutschen Volkes.

\section{REFERENCES}

Alving, B. O. (1968). Spontaneous activity in isolated somata of Aplysia pacemaker neurons. F. gen. Physiol. 51, $29-45$.

Arvanttaki, A. \& Chalazonitis, N. (1967). Electrical properties and temporal organization in oscillatory neurons. In Neurobiology of Invertebrates, (ed. J. Salánki), pp. 169-199. Budapest: Akademiai Kiado.

Ayers, J. L. Jr. \& Selverston, A. I. (1979). Monosynaptic entrainment of an endogenous pacemaker network: a cellular mechanism of von Holst's magnet effect. J. comp. Physiol. 129, 5-17.

BenJAmin, P. R. (1978). Endogenous and synaptic factors affecting the bursting of double spiking molluscan neurosecretory neurons. In Abnomal Neumnal Discharges, (eds N. Chalazonitis \& M. Boisson). New York: Raven Press.

Benjamin, P. R. \& Rose, R. M. (1980). Interneuronal circuitry underlying cyclical feeding in gastropod molluscs. TINS 3, 272-274.

BENNETT, M. V. L. (1977). Electrical transmission: a functional analysis and comparison to chemical transmission. In Handbook of Physiology, Vol. 2, (ed. E. R. Kandel). Maryland: Bethesda.

BENSON, J. A. (1980). Burst reset and frequency control of the neuronal oscillators in the cardiac ganglion of the crab, Portunus sanguinolentus. J. exp. Biol. 87, 285-313.

CARriker, M. R. (1946). Morphology of the alimentary system of the snail Lymnaea stagnalis apressa Say. Trans. Wis. Acad. Sci. Arts Lett. 38, 1-88.

Chen, C. F., von Baumgarten, R. \& Takeda, R. (1971). Pacemaker properties of completely isolated neurones in Aplysia californica. Nature, Lond. 233, 27-29.

Egelhaf, M. (1981a). Coupled neuronal oscillators in the snail Lymnaea stagnalis: endogenous cellular properties and network interactions. Diplom thesis, Universitłt Tübingen, Federal Republic of Germany.

EgelhaA, M. $(1981 b)$. Network interactions of electrotonically coupled neuronal oscillators. Verh. $d t$. zool. Ges. 1981, 164.

Egelhas, M. \& Benjamin, P. R. (1982). Inhibition by recurrent excitation: a mechanism for spike synchronization in a network of coupled neuronal oscillators. $\mathcal{F}$. exp. Biol. $96,447-452$.

GetTing, P. A. (1974). Modification of neuron properties by electrotonic synapses. I. Input resistance, time constant and integration. $\mathcal{F}$. Neurophysiol. 37, 846-857.

Kaneko, C. R., Merickel, M. \& Kater, S. B. (1978). Centrally programmed feeding in Helisoma: identification and characteristics of an electrically coupled premotor neuron network. Brain Res. 146, 1-21.

Kostenko, M. A., Geletyuk, V. I. \& Vepruntsev, B. N. (1974). Completely isolated neurons in the mollusc Lymnaea stagnalis. A new objective for nerve cell biology investigation. Comp. Biochem. Physiol. 49, 89-100.

London, J. A. \& Merickel, M. (1979). Single cell isolation: A way to examine network interactions. Brain Res. 179, 219-230.

MAYERI, E. (1973a). Functional organization of the cardiac ganglion of the lobster, Homaras americanus. $\mathcal{f}$. gen. Physiol. 62, 448-472. 
MAYERI, E. (1973b). A relaxation oscillator description of the burst-generating mechanism in the cardiac ganglion of the lobster, Homaras americanus. J. gen. Physiol. 62, 473-488.

McCrohan, C. R. \& Benjamin, P. R. (1980). Patterns of activity and axonal projections of the Cerebral Giant Cells of the snail, Lymnaea stagnalis. F. exp. Biol. 85, 149-168.

Merickel, M. B., Enman, E. D. \& Kater, S. B. (1977). Analysis of a network of electrically coupled neurons producing rhythmic activity in the snail Helisoma trivolvis. IEEE Trans. biomed. Eng. 24, 277-287.

Mulloney, B., Perkel, D. H. \& Budelli, R. W. (1981). Motor-pattern production: interaction of chemical and electrical synapses. Brain Res. 229, 25-33.

Nicholls, J. G. \& BAYLor, D. A. (1968). Specific modalities and receptive fields of sensory neurones in CNS of the leech. F. Neurophysiol. 31, 740-756.

Pavlidis, T. (1973). Biological Oscillators: Their Mathematical Analysis. New York: Academic Press.

Pinsker, H. M. (1977a). Aplysia bursting neurons as endogenous oscillators. I. Phase-response curves for pulsed inhibitory synaptic input. $\mathcal{F}$. Neumphysiol. 40, 527-543.

Pinsrer, H. M. (1977b). Aplysia bursting neurons as endogenous oscillators. II. Synchronization and entrainment by pulsed inhibitory synaptic input. 7 . Neurophysiol. 40, 544-556.

Pinsker, H. M. \& KANDel, E. R. (1977). Short-term modulation of endogenous bursting rhythms by monosynaptic inhibition in Aplysia neurons: Effects of contingent stimulation. Brain Res. 125, 51-64.

Selverston, A. I. (1980). Are central pattern generators understandable? Behavl. Brain Sci. 3, 535-571.

Selverston, A. I., Russel, D. F., Miller, J. P. \& King, D. G. (1976). The stomatogastric nervous system: structure and function of a small neural network. Progr. Neurobiol. 7, 215-290.

Stent, G. S., Krigtan, W. B., Fruesen, W. O., Ort, C. A. I., Poon, M. \& Calabrese, R. L. (1978). Neuronal generation of the leech swimming movement, Science, N.Y. 200, 1348-1357.

STRumwasser, F. (1967). Types of information stored in single neurons. In Invertebrate Nervous Systems, (ed. C. A. G. Wiersma). Chicago: University of Chicago Press.

Swigchem, H. van (1981). Electrotonic coupling within a cluster of neurosecretory endogenous oscillators in Lymnaea stagnalis (L.) Comp. Biochem. Physiol. 68A, 199-209.

TAZAKI, K. \& CoOKE, I. M. (1979). Spontaneous electrical activity and interaction of large and small cells in cardiac ganglion of the crab, Prtunus sanguinolentus. F. Neurophysiol. 42, 975-999.

Ypey, D. L., Clapham, D. E. \& DeHaan, R. L. (1979). Development of electrical coupling and action potential synchrony between paired aggregates of embryonic heart cells. Membrane Biol. 51, 75-96.

Y PEY, D. L., VANMeerwijK, W. P. M., INCE, C. \& Groos, G. (1980). Mutual entrainment of two pacemaker cells. A study with an electronic parallel conductance model. $\mathcal{f}$. theoret. Biol. 86, 731-755. 